MATHEMATICS OF COMPUTATION

Volume 75 , Number 254 , Pages 697-709

S 0025-5718(05)01807-7

Article electronically published on December 8, 2005

\title{
COMPUTATION OF DIFFERENTIAL OPERATORS IN WAVELET COORDINATES
}

\author{
TSOGTGEREL GANTUMUR AND ROB STEVENSON
}

\begin{abstract}
In [Found. Comput. Math., 2 (2002), pp. 203-245], Cohen, Dahmen, and DeVore proposed an adaptive wavelet algorithm for solving general operator equations. Assuming that the operator defines a boundedly invertible mapping between a Hilbert space and its dual, and that a Riesz basis of wavelet type for this Hilbert space is available, the operator equation is transformed into an equivalent well-posed infinite matrix-vector system. This system is solved by an iterative method, where each application of the infinite stiffness matrix is replaced by an adaptive approximation. It was shown that if the errors of the best linear combinations from the wavelet basis with $N$ terms are $\mathcal{O}\left(N^{-s}\right)$ for some $s>0$, which is determined by the Besov regularity of the solution and the order of the wavelet basis, then approximations yielded by the adaptive method with $N$ terms also have errors of $\mathcal{O}\left(N^{-s}\right)$. Moreover, their computation takes only $\mathcal{O}(N)$ operations, provided $s<s^{*}$, with $s^{*}$ being a measure of how well the infinite stiffness matrix with respect to the wavelet basis can be approximated by computable sparse matrices. Under appropriate conditions on the wavelet basis, for both differential and singular integral operators and for the relevant range of s, in [SIAM J. Math. Anal., 35(5) (2004), pp. 1110-1132] we showed that $s^{*}>s$, assuming that each entry of the stiffness matrix is exactly available at unit cost.

Generally these entries have to be approximated using numerical quadrature. In this paper, restricting ourselves to differential operators, we develop a numerical integration scheme that computes these entries giving an additional error that is consistent with the approximation error, whereas in each column the average computational cost per entry is $\mathcal{O}(1)$. As a consequence, we can conclude that the adaptive wavelet algorithm has optimal computational complexity.
\end{abstract}

\section{INTRODUCTION}

For a Hilbert space $\mathcal{H}$ with dual $\mathcal{H}^{\prime}$, a boundedly invertible linear operator $L: \mathcal{H} \rightarrow \mathcal{H}^{\prime}$, and a $g \in \mathcal{H}^{\prime}$, we consider the problem of finding the solution $u \in \mathcal{H}$ of

$$
L u=g .
$$

In this paper, we will think of this problem as being the result of a variational formulation of an elliptic boundary value problem of order $2 t$ on a domain $\Omega \subset \mathbb{R}^{n}$, so

Received by the editor August 30, 2004 and, in revised form, February 22, 2005.

2000 Mathematics Subject Classification. Primary 41A25, 47A20, 65F50, 65N30, 65D30.

Key words and phrases. Wavelets, matrix compression, differential operators, adaptivity, numerical integration.

This work was supported by the Netherlands Organization for Scientific Research and by the EC-IHP project "Breaking Complexity". 
that $\mathcal{H}$ will be the Sobolev space $H^{t}(\Omega)$, or a closed subspace of that incorporating essential boundary conditions on (a part of) the boundary. With $\Psi=\left\{\psi_{\lambda}: \lambda \in \Lambda\right\}$ being a Riesz basis for $\mathcal{H}$, an equivalent infinite matrix-vector problem reads as

$$
\mathbf{M u}=\mathbf{g},
$$

where, with $\langle$,$\rangle denoting the duality product on \mathcal{H} \times \mathcal{H}^{\prime}, \mathbf{M}:=\langle\Psi, L \Psi\rangle: \ell_{2}(\Lambda) \rightarrow$ $\ell_{2}(\Lambda)$ is boundedly invertible, $\mathbf{g}:=\langle\Psi, g\rangle \in \ell_{2}(\Lambda)$, and $u=\mathbf{u}^{T} \Psi$.

Considering $\Psi$ to be a wavelet basis, in [CDD02 an iterative adaptive method has been developed for approximating the solution of this infinite-dimensional problem by a finitely supported vector within any given tolerance. Roughly speaking, the method consists of an inexact application of a simple iterative scheme to the infinite matrix-vector problem, like damped Richardson in case the infinite stiffness matrix $\mathbf{M}$ is symmetric positive definite. Indeed, since generally the exact application of $\mathbf{M}$ to a current iteration vector already after one iteration would result in an infinitely supported vector, this matrix-vector product has to be approximated. Any column of $\mathbf{M}$ that corresponds to a nonzero entry in the current iteration vector is replaced by a finitely supported approximation within a tolerance that decreases as a function of the size of this coefficient, in such a way that the total error of the resulting approximate matrix product is sufficiently small to retain convergence. Besides this adaptive, inexact matrix-vector multiplication, the other crucial ingredient of the method is a clean-up step that is applied after every fixed number of iterations to remove small coefficients from the current iteration vector in order to control the vector length in relation to the accuracy. To assess the quality of the method, the $\ell_{2}(\Lambda)$-error of the obtained approximation after spending $\mathcal{O}(N)$ operations is compared with that of a best $N$-term approximation for $\mathbf{u}$, i.e., a vector $\mathbf{u}_{N}$ with at most $N$ nonzero coefficients that has $\ell_{2}(\Lambda)$-distance to $\mathbf{u}$ less than or equal to that of any vector with a support of that size.

In any case for wavelets that are sufficiently smooth, the theory of nonlinear approximation ([DeV98, Coh00] $)$ shows that if both

$$
0<s<\frac{d-t}{n}
$$

where $d$ is the order of the wavelets, and the solution $u$ is in the Besov space $B_{\tau}^{s n+t}\left(L_{\tau}(\Omega)\right)$ with $\tau=\left(\frac{1}{2}+s\right)^{-1}$, then $\mathbf{u} \in \mathcal{A}^{s}$, meaning that

$$
\sup _{N \in \mathbb{N}} N^{s}\left\|\mathbf{u}-\mathbf{u}_{N}\right\|<\infty .
$$

Here \|\| denotes the standard norm on $\ell_{2}(\Lambda)$, and later, on other occasions, the standard norm on the space of linear operators from $\ell_{2}(\Lambda)$ to $\ell_{2}(\Lambda)$. Note that for any $\mathbf{v} \in \ell_{2}(\Lambda),\left\|u-\mathbf{v}^{T} \Psi\right\|_{H^{t}} \approx\|\mathbf{u}-\mathbf{v}\|$. In order to avoid the repeated use of generic but unspecified constants, in this paper by $C \lesssim D$ we mean that $C$ can be bounded by a multiple of $D$, independently of parameters that $C$ and $D$ may depend on. Obviously, $C \gtrsim D$ is defined as $D \lesssim C$, and $C \approx D$ as $C \lesssim D$ and $C \gtrsim D$. The attractive feature of these best $N$-term approximations is the fact that the condition involving Besov regularity is much milder than the corresponding condition $u \in H^{s n+t}(\Omega)$ involving Sobolev regularity that would be needed to guarantee the same rate of convergence with approximation from the fixed, i.e., nonadaptive spaces spanned by $N$ wavelets on the coarsest scales.

The efficiency of the adaptive method from CDD02 hinges on the cost of the approximate matrix-vector product, which depends on how well $\mathbf{M}$ can be approximated by a computable sparse matrix. The fact that $\mathbf{M}$ can be expected to be close 
to a sparse matrix is a consequence of the properties of wavelets as being smooth functions and having vanishing moments. We will use the following definition.

Definition 1.1. $M$ is called $s^{*}$-computable, when for each $j \in \mathbb{N}_{0}$, we can construct an infinite matrix $\mathbf{M}_{j}^{*}$ having in each column $\mathcal{O}\left(2^{j}\right)$ nonzero entries, whose computation takes $\mathcal{O}\left(2^{j}\right)$ operations, such that for any $s<s^{*},\left\|\mathbf{M}-\mathbf{M}_{j}^{*}\right\| \lesssim 2^{-j s}$.

The main theorem from CDD02 now says that if $\mathbf{u} \in \mathcal{A}^{s}$ for some $s$, and $\mathbf{M}$ is $s^{*}$-computable for an $s^{*}>s$, then the number of arithmetic operations and storage locations used by the adaptive wavelet algorithm for computing an approximation for $\mathbf{u}$ within tolerance $\varepsilon$ is of the order $\varepsilon^{-1 / s}$. Since in view of (1.2) the same order of storage locations is generally needed to approximate $\mathbf{u}$ within this tolerance using best $N$-term approximations, assuming these would be available, this result shows that this solution method has optimal computational complexity.

Remark 1.2. Actually, instead of being $s^{*}$-computable, in CDD02 it was assumed that $\mathbf{M}$ is " $s^{*}$-compressible". Apart from our addition that each column of $\mathbf{M}_{j}^{*}$ should not only have $\mathcal{O}\left(2^{j}\right)$ entries, but also that, on average, the computation of each of these entries should take $\mathcal{O}(1)$ operations, it is easily seen that the definition of " $s^{*}$-compressible" from CDD02 is equivalent to our definition of $s^{*}$ computable (cf. [Ste03, Remark 2.4]). In CDD02 the average unit cost assumption was mentioned separately afterwards (in Assumption 2).

To conclude optimality of the adaptive wavelet method, it is necessary to show that $\mathbf{M}$ is $s^{*}$-computable for some $s^{*} \geq \frac{d-t}{n}$, since otherwise for a solution $u$ that has sufficient Besov regularity, the computability will be the limiting factor. On the other hand, since, for wavelets of order $d$, by imposing whatever smoothness conditions $\mathbf{u} \in \mathcal{A}^{s}$ can only be guaranteed for $s \leq \frac{d-t}{n}$, showing $s^{*}$-computability for some $s^{*}>\frac{d-t}{n}$ is also a sufficient condition for optimality of the adaptive wavelet method.

Assuming the average unit cost property, $s^{*}$-computability for some $s^{*}>\frac{d-t}{n}$ has been demonstrated in Ste03] for both differential and singular integral operators, and piecewise polynomial wavelets that are sufficiently smooth and have sufficiently many vanishing moments. More precisely, under such conditions it was proven that for some $s^{*}>\frac{d-t}{n}$, the infinite stiffness matrix $\mathbf{M}$ is $s^{*}$-compressible, a concept that, different than in CDD02, we define as follows.

Definition 1.3. $\mathbf{M}$ is called $s^{*}$-compressible, when for each $j \in \mathbb{N}_{0}$, there exists an infinite matrix $\mathbf{M}_{j}$, constructed by dropping entries from $\mathbf{M}$, such that in each column it has $\mathcal{O}\left(2^{j}\right)$ nonzero entries, and such that for any $s<s^{*},\left\|\mathbf{M}-\mathbf{M}_{j}\right\| \lesssim$ $2^{-j s}$.

Only in the special case of a differential operator with constant coefficients, entries of $\mathbf{M}$ can be computed exactly, in $\mathcal{O}(1)$ operations, so that $s^{*}$-compressibility immediately implies $s^{*}$-computability. In general, numerical quadrature is required to approximate the entries. In this paper, considering differential operators, we will show that $\mathbf{M}$ is $s^{*}$-computable for the same value of $s^{*}$ as it was shown to be $s^{*}$-compressible. The case of singular integral operators will be treated in a forthcoming paper (GS05]). We split the task into two parts. First we derive a criterion on the accuracy-work balance of a numerical quadrature scheme to approximate any entry of $\mathbf{M}$, such that, for a suitable choice of the work invested 
in approximating the entries of the compressed matrix $\mathbf{M}_{j}$ as function of both wavelets involved, we obtain an approximation $\mathbf{M}_{j}^{*}$ of which the computation of each column requires $\mathcal{O}\left(2^{j}\right)$ operations, and $\left\|\mathbf{M}_{j}-\mathbf{M}_{j}^{*}\right\| \leq 2^{-j s^{*}}$, meaning that $\mathbf{M}$ is $s^{*}$-computable. Second, we show that we can fulfill the above criterion by the application of standard composite quadrature rules of a fixed, sufficiently high order.

This paper is organized as follows. We collect some error estimates for numerical quadrature in Section 2, In Section 3, assumptions are formulated on the boundary value problem and the wavelets, and the result concerning $s^{*}$-compressibility is recalled from Ste04a. In Section 4, rules for the numerical approximation of the entries of the stiffness matrix are derived, with which $s^{*}$-computability for some $s^{*}>\frac{d-t}{n}$ will be demonstrated.

At the end of this introduction, we fix some more notation. A monomial of $n$ variables is conveniently written using a multi-index $\alpha \in \mathbb{N}_{0}^{n}$ as $x^{\alpha}:=x_{1}^{\alpha_{1}} \cdots x_{n}^{\alpha_{n}}$. Likewise we write partial differentiation operators, that is, $\partial^{\alpha}:=\partial_{1}^{\alpha_{1}} \cdots \partial_{n}^{\alpha_{n}}$. We set $|\alpha|:=\alpha_{1}+\cdots+\alpha_{n}$, and the relation $\alpha \leq \beta$ is defined as $\alpha_{i} \leq \beta_{i}$ for all $i \in\{1, \ldots, n\}$. We have $|\alpha \pm \beta|=|\alpha| \pm|\beta|$ provided that $\alpha-\beta \in \mathbb{N}_{0}^{n}$ in the case of subtraction.

\section{ERror ESTIMATES FOR NUMERICAL QUADRATURE}

We start with deriving an error bound in the $L_{\infty}$-norm for polynomial approximation, which improves upon available results (e.g., in [DL04, Theorem 1.1]) in the sense that our upper bound does not contain an unspecified constant that may vary as a function of the polynomial order $p$. This latter fact will be particularly important for analyzing the errors of quadrature schemes with varying orders as we will apply in [GS05.

Recall that a domain $\Omega$ is called star-shaped with respect to $y \in \Omega$, when the line segment from $y$ to any point in $\Omega$ is contained in $\Omega$, and that it is called star-shaped when it is star-shaped with respect to some $y \in \Omega$. The radius of a star-shaped domain $\Omega$ is defined by

$$
\operatorname{rad}(\Omega):=\min _{y \in S(\Omega)} \max _{x \in \partial \Omega}|x-y|,
$$

where $S(\Omega):=\operatorname{clos}\{y \in \Omega: \Omega$ is star-shaped w.r.t. $y\}$. Apparently, we always have $\operatorname{rad}(\Omega) \leq \operatorname{diam}(\Omega) \leq 2 \operatorname{rad}(\Omega)$, and the radius of a convex domain equals the radius of its smallest circumscribed sphere.

Lemma 2.1. Let $\Omega \subset \mathbb{R}^{n}$ be a star-shaped domain and let $f \in W_{\infty}^{p}(\Omega), p \in \mathbb{N}$. Then there exists a polynomial $g \in P_{p-1}$ on $\Omega$ for which

$$
\|f-g\|_{L_{\infty}(\Omega)} \leq \frac{n^{p}}{p !} \cdot \operatorname{rad}(\Omega)^{p} \cdot|f|_{W_{\infty}^{p}(\Omega)} .
$$

Proof. We first assume that $f \in C^{\infty}(\Omega) \cap W_{\infty}^{p}(\Omega)$. Let a point $y \in S(\Omega)$ be such that $\max _{x \in \partial \Omega}|x-y|=\operatorname{rad}(\Omega)$. Let $g$ be the Taylor polynomial of order $p$ at the point $y$, i.e.,

$$
g(x)=\sum_{|\alpha|<p} \frac{(x-y)^{\alpha}}{\alpha !}\left(\partial^{\alpha} f\right)(y) .
$$


Then the Taylor remainder is given by

$$
f(x)-g(x)=p \sum_{|\alpha|=p} \frac{(x-y)^{\alpha}}{\alpha !} \int_{0}^{1} s^{p-1}\left(\partial^{\alpha} f\right)(x+(y-x) s) d s .
$$

Using

$$
\left|\int_{0}^{1} s^{p-1}\left(\partial^{\alpha} f\right)(x+(y-x) s) d s\right| \leq \int_{0}^{1} s^{p-1} d s \cdot|f|_{W_{\infty}^{p}(\Omega)}=\frac{1}{p} \cdot|f|_{W_{\infty}^{p}(\Omega)}
$$

and

$$
\left|(x-y)^{\alpha}\right|=\left|x_{1}-y_{1}\right|^{\alpha_{1}} \cdots\left|x_{n}-y_{n}\right|^{\alpha_{n}} \leq \operatorname{rad}(\Omega)^{p}
$$

we have

$$
|f(x)-g(x)| \leq \sum_{|\alpha|=p} \frac{1}{\alpha !} \cdot \operatorname{rad}(\Omega)^{p} \cdot|f|_{W_{\infty}^{p}(\Omega)} .
$$

Then by applying the identity

$$
\sum_{|\alpha|=p} \frac{1}{\alpha !}=\frac{n^{p}}{p !}
$$

we get (2.2) for $f \in C^{\infty}(\Omega) \cap W_{\infty}^{p}(\Omega)$.

To complete the proof we use a density argument that was proven in Bur98. For any $f \in W_{\infty}^{p}(\Omega)$, there exist functions $f_{k} \in C^{\infty}(\Omega) \cap W_{\infty}^{p}(\Omega), k \in \mathbb{N}$, such that $f_{k} \rightarrow f$ in $W_{\infty}^{p-1}(\Omega)$, and $\left\|f_{k}\right\|_{W_{\infty}^{p}(\Omega)} \rightarrow\|f\|_{W_{\infty}^{p}(\Omega)}$ as $k \rightarrow \infty$. With this result, for each $k \in \mathbb{N}$ let us denote by $g_{k} \in P_{p-1}$ the Taylor polynomial (2.3) corresponding to $f_{k}$. Then, since for any $k, j \in \mathbb{N}$ and $|\alpha|<p$ we have

$$
\begin{aligned}
\left|\left(\partial^{\alpha} f_{k}\right)(y)-\left(\partial^{\alpha} f_{j}\right)(y)\right| & \leq\left\|\partial^{\alpha} f_{k}-\partial^{\alpha} f_{j}\right\|_{L_{\infty}(\Omega)} \\
& \leq\left\|\partial^{\alpha} f_{k}-\partial^{\alpha} f\right\|_{L_{\infty}(\Omega)}+\left\|\partial^{\alpha} f_{j}-\partial^{\alpha} f\right\|_{L_{\infty}(\Omega)},
\end{aligned}
$$

where the right-hand side tends to zero as $j, k \rightarrow \infty$, we infer that there is a $g \in P_{p-1}$ such that $g_{k} \rightarrow g$ in $L_{\infty}(\Omega)$. Writing

$$
\|f-g\|_{L_{\infty}(\Omega)} \leq\left\|f-f_{k}\right\|_{L_{\infty}(\Omega)}+\left\|g-g_{k}\right\|_{L_{\infty}(\Omega)}+\left\|f_{k}-g_{k}\right\|_{L_{\infty}(\Omega)},
$$

and by taking the limit $k \rightarrow \infty$, the proof is completed.

On a star-shaped domain $\Omega$, let us now consider quadrature rules of the form $Q: f \mapsto \sum_{j} w_{j} f\left(x_{j}\right)$ to approximate $I: f \mapsto \int_{\Omega} f$. We will only consider rules that are internal meaning that all $x_{j} \in \operatorname{clos} \Omega$. The quadrature error functional is defined as $E:=I-Q$.

Proposition 2.2. For a rule $Q$ of order $p$, meaning that $E(f)=0$ for all $f \in$ $P_{p-1}(\Omega)$, and any $f \in W_{\infty}^{p}(\Omega)$ we have

$$
|E(f)| \leq\left(1+\frac{\sum_{j}\left|w_{j}\right|}{\operatorname{vol}(\Omega)}\right) \cdot \frac{n^{p}}{p !} \cdot \operatorname{rad}(\Omega)^{p} \cdot \operatorname{vol}(\Omega) \cdot|f|_{W_{\infty}^{p}(\Omega)} .
$$

Proof. Taking $g$ as in Lemma 2.1, the proof is an easy consequence of that lemma and the estimate

$$
|I(f)-Q(f)|=|I(f)-Q(f)+Q(g)-I(g)| \leq|I(f-g)|+|Q(g-f)| .
$$


Note that for a rule that is positive, meaning that all $w_{j}>0$, and that has order $p>0$, we have $\frac{\sum_{j}\left|w_{j}\right|}{\operatorname{vol}(\Omega)}=1$.

Let us now consider a collection $\mathcal{O}$ of disjoint star-shaped Lipschitz subdomains $\Omega^{\prime} \subset \Omega$, the latter not necessarily being star-shaped, such that clos $\Omega=$ $\bigcup_{\Omega^{\prime} \in \mathcal{O}} \operatorname{clos} \Omega^{\prime}$, which collection we will refer to as being a quadrature mesh. Writing $I(f)$ as $\sum_{\Omega^{\prime} \in \mathcal{O}} \int_{\Omega^{\prime}} f$, on each subdomain $\Omega^{\prime}$ we employ a quadrature rule $Q_{\Omega^{\prime}}(f)=\sum_{j} w_{j}^{\Omega^{\prime}} f\left(x_{j}^{\Omega^{\prime}}\right)$ of order $p$, defining a composite quadrature rule $Q$ of $\operatorname{rank} N:=\# \mathcal{O}$ (and order $p$ ) by $Q(f):=\sum_{\Omega^{\prime} \in \mathcal{O}} Q_{\Omega^{\prime}}(f)$.

Proposition 2.3. For the error functional $E=I-Q$ of this composite quadrature rule, and $f \in W_{\infty}^{p}(\Omega)$ we have

$$
\begin{aligned}
|E(f)| \leq & \left(1+\sup _{\Omega^{\prime} \in \mathcal{O}} \frac{\sum_{j}\left|w_{j}^{\Omega^{\prime}}\right|}{\operatorname{vol}\left(\Omega^{\prime}\right)}\right) \cdot \sup _{\Omega^{\prime} \in \mathcal{O}}\left(\frac{N^{1 / n} \operatorname{rad}\left(\Omega^{\prime}\right)}{\operatorname{diam}(\Omega)}\right)^{p} \\
& \times N^{-p / n} \cdot \frac{n^{p}}{p !} \cdot \operatorname{diam}(\Omega)^{p} \cdot \operatorname{vol}(\Omega) \cdot|f|_{W_{\infty}^{p}(\Omega)} .
\end{aligned}
$$

Proof. Writing $\operatorname{rad}\left(\Omega^{\prime}\right)=\frac{N^{1 / n} \operatorname{rad}\left(\Omega^{\prime}\right)}{\operatorname{diam}(\Omega)} N^{-1 / n} \operatorname{diam}(\Omega)$, and using that $\sum_{\Omega^{\prime} \in \mathcal{O}} \operatorname{vol}\left(\Omega^{\prime}\right)$ $=\operatorname{vol}(\Omega)$, the result follows from Proposition 2.2 .

In view of the above estimate, as well as to control the number of function evaluations that are required, in this paper we will consider families $\left(\mathcal{O}_{\ell}\right)_{\ell \in \mathbb{N}}$ of quadrature meshes and corresponding families of composite quadrature rules $Q_{\ell}$ : $f \mapsto \sum_{Q^{\prime} \in \mathcal{O}_{\ell}} \sum_{j} w_{j}^{\Omega^{\prime}} f\left(x_{j}^{\Omega^{\prime}}\right)$ of rank $N_{\ell}:=\# \mathcal{O}_{\ell}$ and fixed order $p$ that are admissible meaning that they satisfy

$$
\sup _{\ell \in \mathbb{N}, \Omega^{\prime} \in \mathcal{O}_{\ell}} \max \left\{\frac{\sum_{j}\left|w_{j}^{\Omega^{\prime}}\right|}{\operatorname{vol}\left(\Omega^{\prime}\right)}, \frac{N_{\ell}^{1 / n} \operatorname{rad}\left(\Omega^{\prime}\right)}{\operatorname{diam}(\Omega)}, \# x_{j}^{\Omega^{\prime}}\right\}<\infty .
$$

Note that the bound on the number of abscissae in each subdomain is reasonable because the space of polynomials of total degree $p-1$ has $\left(\begin{array}{c}p-1+n \\ n\end{array}\right) \leq p^{n} \lesssim 1$ degrees of freedom.

Finally in this section, we consider product quadrature rules which are generally applied on Cartesian product domains. Let $A$ and $B$ be domains of possibly different dimensions, equipped with the quadrature rules $Q^{(A)}: g \mapsto \sum_{j} w_{j} g\left(x_{j}\right)$ and $Q^{(B)}$ : $h \mapsto \sum_{k} v_{k} h\left(y_{k}\right)$ to approximate $I^{(A)}: g \mapsto \int_{A} g$ and $I^{(B)}: h \mapsto \int_{B} h$, respectively. For simplicity, in this setting we will always assume that these rules are positive and have strictly positive orders. Now with the product rule $Q^{(A)} \times Q^{(B)}$ we mean the mapping $f \mapsto \sum_{j k} w_{j} v_{k} f\left(x_{j}, y_{k}\right)$ to approximate $I: f \mapsto \int_{A \times B} f$.

Lemma 2.4. With error functionals $E^{(A)}:=I^{(A)}-Q^{(A)}$ and $E^{(B)}:=I^{(B)}-Q^{(B)}$, the product rule $Q:=Q^{(A)} \times Q^{(B)}$ satisfies

$$
|I(f)-Q(f)| \leq \operatorname{vol}(A) \sup _{x \in A}\left|E^{(B)}(f(x, \cdot))\right|+\operatorname{vol}(B) \sup _{y \in B}\left|E^{(A)}(f(\cdot, y))\right|,
$$

as long as both $E^{(A)}(f(\cdot, y))$ and $E^{(B)}(f(x, \cdot))$ make sense for all $y \in B$ and $x \in A$, respectively. 
Proof. We have

$$
\begin{aligned}
I(f)-Q(f)= & \int_{A \times B} f(x, y) d x d y-\sum_{j, k} w_{j} v_{k} f\left(x_{j}, y_{k}\right) \\
= & \int_{B}\left(\int_{A} f(x, y) d x-\sum_{j} w_{j} f\left(x_{j}, y\right)\right) d y \\
& +\sum_{j} w_{j}\left(\int_{B} f\left(x_{j}, y\right) d y-\sum_{k} v_{k} f\left(x_{j}, y_{k}\right)\right) .
\end{aligned}
$$

The proof is completed by taking absolute values and using that $\sum_{j} w_{j}=\operatorname{vol}(A)$.

As an application of this lemma, we have the following result for product quadrature rules on rectangular domains.

Proposition 2.5. Consider the rectangular domain $\square:=\left(0, l_{1}\right) \times \cdots \times\left(0, l_{n}\right)$. For the $i$-th coordinate direction, let $Q_{N_{i}}^{(i)}$ be a composite quadrature rule of order $p$ with respect to a quadrature mesh on $\left(0, l_{i}\right)$ of $N_{i}$ equally sized subintervals. Then for the product quadrature rule $Q:=Q_{N_{1}}^{(1)} \times \cdots \times Q_{N_{n}}^{(n)}$ to approximate $I: f \mapsto \int_{\square} f$, and $f$ such that $\partial_{i}^{p} f \in L_{\infty}(\square), i \in\{1, \ldots, n\}$, we have

$$
|I(f)-Q(f)| \leq \frac{2^{1-p}}{p !} \operatorname{vol}(\square) \cdot \sum_{i=1}^{n} l_{i}^{p} N_{i}^{-p} \cdot \max _{i \in\{1, \ldots, n\}}\left\|\partial_{i}^{p} f\right\|_{L_{\infty}(\square)} .
$$

In particular, this quadrature rule is exact on $Q_{p-1}(\square):=P_{p-1}\left(0, l_{1}\right) \times \cdots \times$ $P_{p-1}\left(0, l_{n}\right)$.

Proof. Using that $\operatorname{rad}\left(0, l_{i}\right)=l_{i} / 2$, Proposition 2.3 shows that for each $i$,

$$
\left|\int_{0}^{l_{i}} g-Q_{N_{i}}^{(i)}(g)\right| \leq \frac{2^{1-p}}{p !} N_{i}^{-p} l_{i}^{p+1}|g|_{W_{\infty}^{p}\left(0, l_{i}\right)} .
$$

Using Lemma 2.4 we arrive at the claim by induction.

Corollary 2.6. For the special case $N_{1}=\cdots=N_{n}=N^{1 / n}$, with $l:=\max _{i} l_{i}$ we have

$$
|I(f)-Q(f)| \leq n \frac{2^{1-p}}{p !} N^{-p / n} \cdot l^{n+p} \cdot \max _{i \in\{1, \ldots, n\}}\left\|\partial_{i}^{p} f\right\|_{L_{\infty}(\square)} .
$$

\section{Compressibility}

For some domain $\Omega \subset \mathbb{R}^{n}, t \in \mathbb{N}_{0}$ and $\Gamma_{D} \subset \partial \Omega$, possibly with $\Gamma_{D}=\emptyset$, let

$$
H_{0, \Gamma^{D}}^{t}(\Omega)=\cos _{H^{t}(\Omega)}\left\{u \in H^{t}(\Omega) \cap C^{\infty}(\Omega): \operatorname{supp} u \cap \Gamma^{D}=\emptyset\right\},
$$

and let $L, L^{\prime}: H_{0, \Gamma^{D}}^{t}(\Omega) \rightarrow\left(H_{0, \Gamma^{D}}^{t}(\Omega)\right)^{\prime}$ be defined by

$$
\left\langle L^{\prime} u, v\right\rangle=\langle u, L v\rangle=\sum_{|\alpha|,|\beta| \leq t}\left\langle\partial^{\alpha} u, a_{\alpha \beta} \partial^{\beta} v\right\rangle,
$$

where $a_{\alpha \beta} \in L_{\infty}(\Omega)$ so that $L, L^{\prime}$ are bounded. Obviously $L, L^{\prime}$ have extensions, which we will also denote by $L, L^{\prime}$, respectively, as bounded operators from $H^{t}(\Omega) \rightarrow H^{-t}(\Omega)$. For completeness, $H^{s}(\Omega)$ for $s<0$ denotes the dual of $H^{-s}(\Omega)$. 
We assume that there exists a $\sigma>0$ such that

$$
L, L^{\prime}: H^{t+\sigma}(\Omega) \rightarrow H^{-t+\sigma}(\Omega) \quad \text { are bounded. }
$$

Sufficient is that for arbitrary $\varepsilon>0$, and all $\alpha, \beta$ with $\min \{|\alpha|,|\beta|\}>t-\sigma$,

$$
a_{\alpha \beta} \in\left\{\begin{array}{cc}
W_{\infty}^{\sigma-t+\min \{|\alpha|,|\beta|\}}(\Omega) & \text { when } \sigma \in \mathbb{N} \\
C^{\sigma-t+\min \{|\alpha|,|\beta|\}+\varepsilon}(\Omega) & \text { when } \sigma \notin \mathbb{N} .
\end{array}\right.
$$

In addition, we assume that the coefficients $a_{\alpha \beta}$ are piecewise smooth, in the sense that there exist $M$ disjoint Lipschitz domains $\Omega_{q}, q \in\{1, \ldots, M\}$, such that $a_{\alpha \beta}$ is smooth on each $\Omega_{q}$, and $\operatorname{clos} \Omega=\bigcup_{q} \operatorname{clos} \Omega_{q}$.

Let

$$
\Psi=\left\{\psi_{\lambda}: \lambda \in \Lambda\right\}
$$

be a Riesz basis for $H_{0, \Gamma^{D}}^{t}(\Omega)$ of wavelet type. The index $\lambda$ encodes both the level, denoted by $|\lambda| \in \mathbb{N}_{0}$, and the location of the wavelet $\psi_{\lambda}$. We will assume that the wavelets are local and piecewise smooth with respect to nested subdivisions in the following sense: we assume that there exists a sequence $\left(\mathcal{O}_{\ell}\right)_{\ell \in \mathbb{N}_{0}}$ of collections $\mathcal{O}_{\ell}=\left\{\Omega_{i}^{\ell}: i \in J^{\ell}\right\}$ of disjoint "uniformly" (in $i$ and $\ell$ ) Lipschitz domains $\Omega_{i}^{\ell}$, with $\operatorname{clos} \Omega=\bigcup_{i \in J^{\ell}} \operatorname{clos} \Omega_{i}^{\ell}$ and

$$
\operatorname{diam}\left(\Omega_{i}^{\ell}\right) \approx 2^{-\ell},
$$

and where each $\Omega_{i}^{\ell}$ is contained in some $\Omega_{q}$, and its closure is the union of the closures of a uniformly bounded number of subdomains from $\mathcal{O}_{\ell+1}$. We assume that for each $\lambda \in \Lambda$ there exists a $J_{\lambda} \subset J^{|\lambda|}$ with

$$
\sup _{\lambda \in \Lambda} \# J_{\lambda}<\infty \text { and } \sup _{\ell \in \mathbb{N}_{0}, i \in J^{\ell}} \#\left\{\lambda:|\lambda|=\ell, i \in J_{\lambda}\right\}<\infty,
$$

such that $\operatorname{supp} \psi_{\lambda}=\bigcup_{i \in J_{\lambda}} \operatorname{clos} \Omega_{i}^{|\lambda|}$, being a connected set, and that on each $\Omega_{i}^{|\lambda|}$, $\psi_{\lambda}$ is smooth with

$$
\sup _{x \in \Omega_{i}^{|\lambda|}}\left|\partial^{\beta} \psi_{\lambda}(x)\right| \lesssim 2^{\left(|\beta|+\frac{n}{2}-t\right)|\lambda|} \quad \text { for } \beta \in \mathbb{N}_{0}^{n} .
$$

For a precise definition of a collection of sets to be a collection of uniformly Lipschitz domains, we refer to [Ste04a, Remark 2.1].

Examples of such wavelets are (the images under smooth mappings of) tensor products of univariate spline wavelets, or finite element wavelets subordinate to a subdivision of the domain into $n$-simplices.

Furthermore, we assume that there exist $\gamma>t, \tilde{d}>-t$ such that for $r \in[-\tilde{d}, \gamma)$, $s<\gamma$,

$$
\|\cdot\|_{H^{r}(\Omega)} \lesssim 2^{\ell(r-s)}\|\cdot\|_{H^{s}(\Omega)}, \quad \text { on } W_{\ell}:=\operatorname{span}\left\{\psi_{\lambda}:|\lambda|=\ell\right\} .
$$

For $r>s$, this is the well-known inverse inequality. For $r<s$, (3.4) is a consequence of the property of wavelets of having vanishing moments, or, more generally, cancellation properties.

Remark 3.1. It is known that the above wavelet assumptions are satisfied by biorthogonal wavelets when the primal and dual spaces have regularity indices $\gamma>t, \tilde{\gamma}>0$ and orders $d>\gamma, \tilde{d}>\tilde{\gamma}$ respectively (cf. [Dah96, DS99c]), the primal spaces consist of "piecewise" smooth functions, and finally, no boundary conditions are imposed on the dual spaces (cf. [DS98]). In particular, (3.4) for $r \in[-\tilde{d},-\tilde{\gamma}]$ can 
be deduced from the lines following (A.2) in DS99c. In case homogeneous boundary conditions are incorporated in the dual spaces, slightly weaker statements can be proven; see [Ste04a, Remark 2.5].

We recall here the main result on compressibility for differential operators from Ste04a].

Theorem 3.2. Let $\mathbf{M}=\langle\Psi, L \Psi\rangle$. Choose $\kappa$ satisfying

$$
\begin{aligned}
& \kappa=\frac{1}{n-1} \\
& \kappa>\frac{\min \{t+\tilde{d}, \sigma\}}{\gamma-t} \quad \text { and } \quad \kappa \geq 1 \quad \text { when } n=1 .
\end{aligned}
$$

For $j \in \mathbb{N}$, define the infinite matrix $\mathbf{M}_{j}$ by replacing all entries $M_{\lambda \lambda^{\prime}}=\left\langle\psi_{\lambda}, L \psi_{\lambda^{\prime}}\right\rangle$ by zeros when

$$
\begin{aligned}
& || \lambda|-| \lambda^{\prime}||>j \kappa, \text { or } \\
& || \lambda|-| \lambda^{\prime}||>j / n \text { and }\left\{\begin{array}{l}
\exists i^{\prime} \in J_{\lambda^{\prime}}, \operatorname{supp} \psi_{\lambda} \subseteq \operatorname{clos} \Omega_{i^{\prime}}^{\left|\lambda^{\prime}\right|} \\
\exists i \in J_{\lambda}, \operatorname{supp} \psi_{\lambda^{\prime}} \subseteq \operatorname{clos} \Omega_{i}^{|\lambda|}|\lambda|>\left|\lambda^{\prime}\right|,
\end{array} \text { when }|\lambda|<\left|\lambda^{\prime}\right| .\right.
\end{aligned}
$$

Then the number of nonzero entries in each column of $\mathbf{M}_{j}$ is of order $2^{j}$, and for any

$$
s \leq \min \left\{\frac{t+\tilde{d}}{n}, \frac{\sigma}{n}\right\}, \text { with } s<\frac{\gamma-t}{n-1} \text { when } n>1,
$$

it follows that $\left\|\mathbf{M}-\mathbf{M}_{j}\right\| \lesssim 2^{-j s}$. We conclude that $\mathbf{M}$ is $s^{*}$-compressible, as defined in Definition 1.3, with $s^{*}=\min \left\{\frac{t+\tilde{d}}{n}, \frac{\sigma}{n}, \frac{\gamma-t}{n-1}\right\}$ when $n>1$, and $s^{*}=\min \{t+\tilde{d}, \sigma\}$ when $n=1$.

From this theorem we infer that if $\tilde{d}>d-2 t, \sigma>d-t$ and, when $n>1$, $\frac{\gamma-t}{n-1}>\frac{d-t}{n}$, then $s^{*}>\frac{d-t}{n}$ as required. For $n>1$, the condition involving $\gamma$ is satisfied for instance for spline wavelets, where $\gamma=d-\frac{1}{2}$, in case $\frac{d-t}{n}>\frac{1}{2}$.

If each entry of $\mathbf{M}$ can be exactly computed in $\mathcal{O}(1)$ operations, then $s^{*}$-compressibility implies $s^{*}$-computability, as defined in Definition 1.1, and so, when indeed $s^{*}>\frac{d-t}{n}$, it implies the optimal computational complexity of the adaptive wavelet scheme from CDD02. This assumption on the computation of the entries is realistic when both the coefficients $a_{\alpha \beta}$ of the differential operator and the wavelets are piecewise polynomials. In general, however, numerical quadrature will be needed to approximate the entries of $\mathbf{M}_{j}$. Then the question arises how to realize a sufficient accuracy of these approximations such that the additional error has, qualitatively, the same upper bound as $\left\|\mathbf{M}-\mathbf{M}_{j}\right\|$, where in each column the average work per entry is $\mathcal{O}(1)$, in which case $s^{*}$-compressibility implies $s^{*}$-computability. In the next section, additionally assuming that the wavelets are essentially piecewise polynomials, we will see that it is possible to select quadrature rules with which this is realized.

\section{Computability}

Let us denote by $\mathbf{M}_{j}^{*}$ the matrix, with elements $M_{j, \lambda \lambda^{\prime}}^{*}$, obtained by approximating the entries of $\mathbf{M}_{j}$ using some numerical scheme dependent on $j$. The following theorem defines a criterion on the computational cost in relation to the accuracy for computing individual entries of $\mathbf{M}$ so that $s^{*}$-compressibility implies $s^{*}$-computability. 
Theorem 4.1. Let $\mathbf{M}, \mathbf{M}_{j}$ and $s^{*}$ be as in Theorem 3.2. Assume that for some $d^{*} \in \mathbb{R}$ and $p$ with

$$
p>s^{*} n+d^{*} \text { and } p \geq s^{*} n,
$$

an approximation $M_{\lambda \lambda^{\prime}}^{*}$ of $M_{\lambda \lambda^{\prime}}$ can be computed in $\mathcal{O}(N)$ operations, having an error

$$
\left|M_{\lambda \lambda^{\prime}}-M_{\lambda \lambda^{\prime}}^{*}\right| \lesssim N^{-p / n} 2^{-|| \lambda|-| \lambda^{\prime}||\left(n / 2+p-d^{*}\right)} .
$$

Then for parameters $\theta$ and $\varrho$ with

$$
\theta \leq 1 \quad \text { and } \quad s^{*} n / p \leq \theta \leq \varrho<1-d^{*} / p,
$$

by spending the number

$$
N_{j, \lambda \lambda^{\prime}} \bar{\sim} \max \left\{1,2^{j \theta-|| \lambda|-| \lambda^{\prime}|| n \varrho}\right\}
$$

of arithmetical operations to the computation of $M_{j, \lambda \lambda^{\prime}}^{*}$, one has $\left\|\mathbf{M}_{j}-\mathbf{M}_{j}^{*}\right\| \lesssim$ $2^{-j s^{*}}$, and the work for computing each column of $\mathbf{M}_{j}^{*}$ is of order $2^{j}$.

Since the conditions (4.1) and (4.3) define a nonempty set in the $(\theta, \varrho)$-plane, we conclude that $\mathbf{M}$ is $s^{*}$-computable.

The proof will use Schur's lemma, which we recall here for the reader's convenience.

Lemma 4.2 (Schur's lemma). If for a matrix $\mathbf{A}=\left(a_{\lambda, \lambda^{\prime}}\right)_{\lambda, \lambda^{\prime} \in \Lambda}$, there is a sequence $\omega_{\lambda}>0, \lambda \in \Lambda$, and a constant $C$ such that

$$
\sum_{\lambda^{\prime} \in \Lambda} \omega_{\lambda^{\prime}}\left|a_{\lambda \lambda^{\prime}}\right| \leq \omega_{\lambda} C \quad(\lambda \in \Lambda) \quad \text { and } \quad \sum_{\lambda \in \Lambda} \omega_{\lambda}\left|a_{\lambda \lambda^{\prime}}\right| \leq \omega_{\lambda^{\prime}} C \quad\left(\lambda^{\prime} \in \Lambda\right),
$$

then $\|\mathbf{A}\| \leq C$.

Proof of Theorem 4.1. Denoting the $\left(\lambda, \lambda^{\prime}\right)$-th entry of the error matrix $\mathbf{M}_{j}-\mathbf{M}_{j}^{*}$ by $\varepsilon_{j, \lambda \lambda^{\prime}}$, from (4.2) and (4.4) we have

$$
\varepsilon_{j, \lambda \lambda^{\prime}} \lesssim N_{j, \lambda \lambda^{\prime}}^{-p / n} 2^{-|| \lambda|-| \lambda^{\prime}||\left(n / 2+p-d^{*}\right)} \lesssim 2^{-\| \lambda|-| \lambda^{\prime}||\left(n / 2+p-\varrho p-d^{*}\right)} 2^{-j \theta p / n} .
$$

We have $\sigma:=n / 2+p-\varrho p-d^{*}=n / 2+p\left(1-\varrho-d^{*} / p\right)>n / 2$ from (4.3). Let $\lambda$ be some given index. The locality assumptions on the wavelets show that for fixed $\lambda \in \Lambda$, the number of indices $\lambda^{\prime}$ with fixed $\left|\lambda^{\prime}\right|$ with $\operatorname{vol}\left(\operatorname{supp} \psi_{\lambda^{\prime}} \cap \operatorname{supp} \psi_{\lambda}\right)>0$ is of order $\max \left\{1,2^{\left(\left|\lambda^{\prime}\right|-|\lambda|\right) n}\right\}$. With weights $\omega_{\lambda^{\prime}}=2^{-\left|\lambda^{\prime}\right| n / 2}$, we find

$$
\begin{aligned}
\omega_{\lambda}^{-1} \sum_{\lambda^{\prime}} \omega_{\lambda^{\prime}}\left|\varepsilon_{j, \lambda \lambda^{\prime}}\right| \lesssim & 2^{|\lambda| n / 2} \sum_{0 \leq\left|\lambda^{\prime}\right| \leq|\lambda|} 2^{-\left|\lambda^{\prime}\right| n / 2} 2^{-\left(|\lambda|-\left|\lambda^{\prime}\right|\right) \sigma} 2^{-j \theta p / n} \cdot 1 \\
& +2^{|\lambda| n / 2} \sum_{\left|\lambda^{\prime}\right|>|\lambda|} 2^{-\left|\lambda^{\prime}\right| n / 2} 2^{-\left(\left|\lambda^{\prime}\right|-|\lambda|\right) \sigma} 2^{-j \theta p / n} \cdot 2^{\left(\left|\lambda^{\prime}\right|-|\lambda|\right) n} \\
\lesssim & 2^{-j \theta p / n} .
\end{aligned}
$$

By the symmetry of the estimate (4.5) in $\lambda$ and $\lambda^{\prime}$, from Schur's lemma we conclude that

$$
\left\|\mathbf{M}_{j}-\mathbf{M}_{j}^{*}\right\| \lesssim 2^{-j \theta p / n} \leq 2^{-j s^{*}}
$$

because $\theta \geq s^{*} n / p$. 
Denoting by $\Lambda_{j, \lambda}$ the set of row indices of nonzero entries in the $\lambda$-th column of $\mathbf{M}_{j}$, the computational work $W_{j, \lambda}$ for this column is

$$
\begin{aligned}
W_{j, \lambda}=\sum_{\lambda^{\prime} \in \Lambda_{j, \lambda}} N_{j, \lambda \lambda^{\prime}} & \lesssim \sum_{\lambda^{\prime} \in \Lambda_{j, \lambda}} \max \left\{1,2^{j \theta-|| \lambda|-| \lambda^{\prime}|| n \varrho}\right\} \\
& \lesssim 2^{j}+\sum_{\left\{\lambda^{\prime} \in \Lambda_{j, \lambda}:|| \lambda|-| \lambda^{\prime}|| \leq j / n\right\}} 2^{j \theta-|| \lambda|-| \lambda^{\prime}|| n \varrho},
\end{aligned}
$$

where we used the fact that, since $\varrho \geq \theta, 2^{j \theta-|| \lambda|-| \lambda^{\prime}|| n \varrho}<1$ for $\| \lambda|-| \lambda^{\prime}||>j / n$, and that the number of nonzero entries in each column of $\mathbf{M}_{j}$ is $\mathcal{O}\left(2^{j}\right)$. The second term can be bounded by a constant multiple of

$$
\begin{aligned}
& \sum_{-j / n \leq\left|\lambda^{\prime}\right|-|\lambda| \leq 0} 2^{j \theta-\left(|\lambda|-\left|\lambda^{\prime}\right|\right) n \varrho} \cdot 1+\sum_{0<\left|\lambda^{\prime}\right|-|\lambda| \leq j / n} 2^{j \theta-\left(\left|\lambda^{\prime}\right|-|\lambda|\right) n \varrho} \cdot 2^{\left(\left|\lambda^{\prime}\right|-|\lambda|\right) n} \\
& \lesssim 2^{j \theta} 2^{j \max \{0,1-\varrho\}} .
\end{aligned}
$$

From (4.3) we have $\theta \leq 1$ and $\theta \leq \varrho$, and so $1-\varrho+\theta \leq 1$, from which we conclude that $W_{j, \lambda}=\mathcal{O}\left(2^{j}\right)$.

By applying the error estimates from Section 2, we will now show how numerical quadrature schemes satisfying (4.2) can be realized. An entry of the matrix can be rewritten as

$$
M_{\lambda \lambda^{\prime}}=\sum_{|\alpha|,|\beta| \leq t} \int_{\operatorname{supp} \psi_{\lambda} \cap \operatorname{supp} \psi_{\lambda^{\prime}}} a_{\alpha \beta} \partial^{\alpha} \psi_{\lambda} \partial^{\beta} \psi_{\lambda^{\prime}}
$$

Without loss of generality, in the remainder of this section we assume that

$$
|\lambda| \geq\left|\lambda^{\prime}\right| \text {. }
$$

Then, it is clear that the intersection $\operatorname{supp} \psi_{\lambda} \cap \operatorname{supp} \psi_{\lambda^{\prime}}$ is the union of sets $\Omega_{i}^{|\lambda|}$, $i \in J_{\lambda \lambda^{\prime}}$, for some $J_{\lambda \lambda^{\prime}} \subseteq J_{\lambda}$. Therefore we can expand the integral into integrals of smooth functions

$$
M_{\lambda \lambda^{\prime}}=\sum_{i \in J_{\lambda \lambda^{\prime}}} I_{\lambda \lambda^{\prime}, i}
$$

where

$$
I_{\lambda \lambda^{\prime}, i}:=\sum_{|\alpha|,|\beta| \leq t} \int_{\Omega_{i}^{|\lambda|}} a_{\alpha \beta} \partial^{\alpha} \psi_{\lambda} \partial^{\beta} \psi_{\lambda^{\prime}} .
$$

Recall that for each $\ell \in \mathbb{N}_{0}, i \in J^{\ell}$, there is a $q=q(\ell, i) \in\{1, \ldots, M\}$ with $\Omega_{i}^{\ell} \subset \Omega_{q}$, and furthermore that all $a_{\alpha \beta}$ are smooth on any $\Omega_{q}$. In the following, we assume that there is a constant $e \in \mathbb{N}$, and that there are smooth regular mappings $\kappa_{q}: \mathbb{R}^{n} \rightarrow \mathbb{R}^{n}$, such that for each $\lambda \in \Lambda, i \in J_{\lambda}$, and $q=q(|\lambda|, i)$,

$$
\left.\left(\psi_{\lambda} \circ \kappa_{q}\right)\right|_{\kappa_{q}^{-1}\left(\Omega_{i}^{|\lambda|}\right)} \in P_{e-1} .
$$

With the commonly used approaches to constructed wavelets on nontrivial geometries via domain decomposition techniques (DS99a, CTU99, CM00, DS99b, Ste04b]), the above assumption is valid when one starts from a piecewise polynomial multiresolution analysis on the corresponding reference domain. Note that the smallest $e$ for which (4.8) holds satisfies $e \geq d(\geq t+1)$.

Since by a transformation of coordinates, $\sum_{|\alpha|,|\beta| \leq t} \int_{\Omega_{i}^{|\lambda|}} a_{\alpha \beta} \partial^{\alpha} \psi_{\lambda} \partial^{\beta} \psi_{\lambda^{\prime}}$ can be written as $\sum_{|\alpha|,|\beta| \leq t} \int_{\kappa_{q}^{-1}\left(\Omega_{i}^{|\lambda|}\right)} \tilde{a}_{\alpha \beta} \partial^{\alpha}\left(\psi_{\lambda} \circ \kappa_{q}\right) \partial^{\beta}\left(\psi_{\lambda^{\prime}} \circ \kappa_{q}\right)$, where, as $a_{\alpha \beta}, \tilde{a}_{\alpha \beta}$ is a 
function that is smooth, in the following without loss of generality we may assume that $\kappa_{q}=$ id.

Proposition 4.3. Consider a composite quadrature rule from an admissible family (uniformly in $\lambda \in \Lambda$ and $i \in J_{\lambda}$ ) of fixed order $p$ and rank $N$ to approximate each of the integrals from (4.7), where $\left.\psi_{\lambda}\right|_{\Omega_{i}^{|\lambda|}} \in P_{e-1}$. Then, with

$$
d^{*}:=e-1-t,
$$

the error of this numerical integration is bounded by

$$
\left|M_{\lambda \lambda^{\prime}}-M_{\lambda \lambda^{\prime}}^{*}\right| \lesssim N^{-p / n} 2^{-\| \lambda|-| \lambda^{\prime}||\left(n / 2+p-d^{*}\right)} .
$$

Taking $p>s^{*} n+d^{*}$, we conclude that the criterion for $s^{*}$-computability from Theorem 4.1 is satisfied.

Proof. In view of Proposition 2.3 we have to bound $\partial^{\zeta}\left(a_{\alpha \beta} \partial^{\alpha} \psi_{\lambda} \partial^{\beta} \psi_{\lambda^{\prime}}\right)$ for $|\zeta|=p$, or $\partial^{\eta} a_{\alpha \beta} \partial^{\alpha+\theta} \psi_{\lambda} \partial^{\beta+\xi} \psi_{\lambda^{\prime}}$ for $|\eta+\theta+\xi|=p$. Since $a_{\alpha \beta}$ is smooth, $|\lambda| \geq\left|\lambda^{\prime}\right|$, and $\partial^{\alpha+\theta} \psi_{\lambda}$ vanishes when $|\alpha+\theta| \geq e$, by invoking (3.3) we see that the worst case occurs when $\eta=0,|\alpha+\theta|=r:=\min \{e-1,|\alpha|+p\}$, and thus $|\xi|=p-r+|\alpha|$, yielding

$$
\begin{aligned}
\left|a_{\alpha \beta} \partial^{\alpha} \psi_{\lambda} \partial^{\beta} \psi_{\lambda^{\prime}}\right|_{W_{\infty}^{p}\left(\Omega_{i}^{|\lambda|}\right)} \lesssim 2^{(r+n / 2-t)|\lambda|} 2^{(p-r+|\alpha|+|\beta|+n / 2-t)\left|\lambda^{\prime}\right|} \\
\leq 2^{(e-1+n / 2-t)|\lambda|} 2^{(p-e+1+|\alpha|+|\beta|+n / 2-t)\left|\lambda^{\prime}\right|} .
\end{aligned}
$$

Now using that $\operatorname{diam}\left(\Omega_{i}^{|\lambda|}\right) \approx 2^{-|\lambda|}$ and $|\alpha|,|\beta| \leq t$, Proposition 2.3 shows that

$$
\begin{aligned}
\left|M_{\lambda \lambda^{\prime}}-M_{\lambda \lambda^{\prime}}^{*}\right| & \lesssim N^{-p / n} 2^{-|\lambda|(n+p)} 2^{(e-1+n / 2-t)|\lambda|} 2^{(p-e+1+t+n / 2)\left|\lambda^{\prime}\right|} \\
& =N^{-p / n} 2^{-|| \lambda|-| \lambda^{\prime}||\left(n / 2+p-d^{*}\right)} .
\end{aligned}
$$

In the case of tensor product constructions yielding wavelets that are piecewise in $Q_{d-1}$, the (piecewise) polynomial order $e$ is $n(d-1)+1$, so that $d^{*}$ from Proposition 4.3 is equal to $n(d-1)-t(\geq(n-1) d)$. In the next proposition, we will see that for such wavelets the application of product quadrature rules gives rise to smaller $d^{*}$, and so allows for smaller quadrature orders $p$.

Proposition 4.4. Suppose that $\Omega_{i}^{|\lambda|}$ is an n-rectangle, $\left.\psi_{\lambda}\right|_{\Omega_{i}^{|\lambda|}} \in Q_{d-1}\left(\Omega_{i}^{|\lambda|}\right)$, and that a product composite quadrature rule of order $p$ and rank $N$ as in Corollary 2.6 is applied to approximate each of the integrals from (4.7). Then, with

$$
d^{*}:=d-1,
$$

the error of the numerical integration is bounded by

$$
\left|M_{\lambda \lambda^{\prime}}-M_{\lambda \lambda^{\prime}}^{*}\right| \lesssim N^{-p / n} 2^{-|| \lambda|-| \lambda^{\prime}||\left(n / 2+p-d^{*}\right)} .
$$

Taking $p>s^{*} n+d^{*}$, we conclude that the criterion for $s^{*}$-computability from Theorem 4.1 is satisfied.

Proof. Without loss of generality, we may assume that the $n$-rectangle $\Omega_{i}^{|\lambda|} \subset \mathbb{R}^{n}$ is aligned with the Cartesian coordinates. In view of Corollary 2.6, for $i \in\{1, \ldots, n\}$ we have to bound $\partial_{i}^{p}\left(a_{\alpha \beta} \partial^{\alpha} \psi_{\lambda} \partial^{\beta} \psi_{\lambda^{\prime}}\right)$, or $\partial_{i}^{k} a_{\alpha \beta} \partial_{i}^{l} \partial^{\alpha} \psi_{\lambda} \partial_{i}^{m} \partial^{\beta} \psi_{\lambda^{\prime}}$ for $k+l+m=p$. Since $a_{\alpha \beta}$ is smooth, $|\lambda| \geq\left|\lambda^{\prime}\right|$, and $\partial_{i}^{l} \partial^{\alpha} \psi_{\lambda}$ vanishes when $\alpha_{i}+l \geq d$, by invoking 
(3.3) we see that the worst case occurs when $k=0, \alpha_{i}+l=r:=\min \left\{d-1, \alpha_{i}+p\right\}$, and thus $m=p-r+\alpha_{i}$, yielding

$$
\begin{aligned}
\left|\partial_{i}^{p}\left(a_{\alpha \beta} \partial^{\alpha} \psi_{\lambda} \partial^{\beta} \psi_{\lambda^{\prime}}\right)\right| & \lesssim 2^{\left(|\alpha|-\alpha_{i}+r+n / 2-t\right)|\lambda|} 2^{\left(p-r+\alpha_{i}+|\beta|+n / 2-t\right)\left|\lambda^{\prime}\right|} \\
& \lesssim 2^{(|\alpha|+d-1+n / 2-t)|\lambda|} 2^{(p-d+1+|\beta|+n / 2-t)\left|\lambda^{\prime}\right|}
\end{aligned}
$$

Since $\operatorname{diam}\left(\Omega_{i}^{|\lambda|}\right) \approx 2^{-|\lambda|}$ and $|\alpha|,|\beta| \leq t$, an application of Corollary 2.6 shows that

$$
\begin{aligned}
\left|M_{\lambda \lambda^{\prime}}-M_{\lambda \lambda^{\prime}}^{*}\right| & \lesssim N^{-p / n} 2^{-|\lambda|(n+p)} 2^{(d-1+n / 2)|\lambda|} 2^{(p-d+1+n / 2)\left|\lambda^{\prime}\right|} \\
& =N^{-p / n} 2^{-|| \lambda|-| \lambda^{\prime}||\left(n / 2+p-d^{*}\right)} .
\end{aligned}
$$

\section{REFERENCES}

[Bur98] V. I. Burenkov, Sobolev spaces on domains. Teubner Verlag, Stuttgart, Leipzig, 1998. MR 1622690 (99g:46040)

[CDD02] A. Cohen, W. Dahmen and R. DeVore. Adaptive wavelet methods II - Beyond the elliptic case. Found. Comput. Math., 2(3):203-245, 2002. MR1907380 (2003f:65212)

[Coh00] A. Cohen. Wavelet methods in numerical analysis. in P.G. Ciarlet and J.L. Lions (eds.), Handbook of numerical analysis. Vol. VII, pp.417-711, North-Holland, Amsterdam, 2000. MR1804747 (2002c:65252)

[CM00] A. Cohen and R. Masson. Wavelet adaptive method for second order elliptic problems: Boundary conditions and domain decomposition. Numer. Math., 86:193-238, 2000. MR:1777487 (2001j:65185)

[CTU99] C. Canuto, A. Tabacco and K. Urban. The wavelet element method part I: Construction and analysis. Appl. Comput. Harmon. Anal., 6:1-52, 1999. MR1664902 (99k:42055)

[Dah96] W. Dahmen. Stability of multiscale transformations. J. Four. Anal. Appl., 4:341-362, 1996. MR1395769(97i:46133)

[DeV98] R. DeVore. Nonlinear approximation. Acta Numer., 7:51-150, 1998. MR.1689432 (2001a:41034)

[DL04] S. Dekel and D. Leviatan. The Bramble-Hilbert lemma for convex domains. SIAM J. Numer. Anal., 35(5):1203-1212, 2004. MR2050198(2005d:41010)

[DS98] W. Dahmen and R. Schneider. Wavelets with complementary boundary conditions - Function spaces on the cube. Results in Math., 34:255-293, 1998. MR 1652724 (99h:42057)

[DS99a] W. Dahmen and R. Schneider. Composite wavelet bases for operator equations. Math. Comput., 68:1533-1567, 1999. MR 1648379 (99m:65122)

[DS99b] W. Dahmen and R. Schneider. Wavelets on manifolds I: Construction and domain decomposition. SIAM J. Math. Anal., 31:184-230, 1999. MR.1742299(2000k:65242)

[DS99c] W. Dahmen and R.P. Stevenson. Element-by-element construction of wavelets satisfying stability and moment conditions. SIAM J. Numer. Anal., 37(1):319-352, 1999. MR 1742747 (2001c:65144)

[GS05] T. Gantumur and R.P. Stevenson. Computation of singular integral operators in wavelet coordinates. Computing, 76:77-107, 2006.

[Ste03] R.P. Stevenson. Adaptive solution of operator equations using wavelet frames. SIAM J. Numer. Anal., 41(3):1074-1100, 2003. MR2005196 (2004e:42062)

[Ste04a] R.P. Stevenson. On the compressibility of operators in wavelet coordinates. SIAM J. Math. Anal., 35(5):1110-1132, 2004. MR2050194 (2005e:42128)

[Ste04b] R.P. Stevenson. Composite wavelet bases with extended stability and cancellation properties. Preprint 1304, Department of Mathematics, Utrecht University, 2004. Submitted.

Department of Mathematics, Utrecht University, P. O. Box 80.010, NL-3508 TA Utrecht, The Netherlands

E-mail address: gantumur@math.uu.nl

Department of Mathematics, Utrecht University, P. O. Box 80.010, NL-3508 TA Utrecht, The Netherlands

E-mail address: stevenson@math.uu.nl 